\title{
An Affected Child with Arrhythmogenic Right Ventricular Dysplasia and Severe Left Ventricle Dysfunction: A Rare Case Report
}

\author{
Ali Akbar Zeinaloo ${ }^{1}$, Ehsan Aghaei Moghadam ${ }^{1}$, Amir Salimi ${ }^{2}$, Azin Ghamari ${ }^{3}$, Helia Mojtabavi ${ }^{1}$, Amir \\ Bahador Golchin ${ }^{1}$ and Shakila Meshkat ${ }^{3, "}$ \\ ${ }^{1}$ Tehran University of Medical Sciences, Tehran, Iran \\ ${ }^{2}$ Shahid Beheshti University of Medical Sciences, Tehran, Iran \\ ${ }^{3}$ Growth and Development Research Center, Tehran University of Medical Science, Tehran, Iran \\ "Corresponding author: Growth and Development Research Center, Tehran University of Medical Science, Tehran, Iran. Tel: +98-9121483681, Email: \\ shakilameshkat1994@gmail.com \\ Received 2019 July 06; Accepted 2019 October 01.
}

\begin{abstract}
Arrhythmogenic Right Ventricular Dysplasia (ARVD) was diagnosed in a nine-year-old boy following the presentation of generalized edema, ascites, and palpitation. Typical signs of ARVD on electrocardiography (ECG) and echocardiography were detected in initial assessments. On echocardiography, severe right ventricle dysfunction, free tricuspid regurgitation, and left ventricle ejection fraction (EF) of $45 \%-50 \%$ were reported. Cardiac magnetic resonance images showed severely enlarged right ventricle size with severely reduced systolic function. Prolonged episodes of sinus bradycardia and atrial fibrillation were noted at each hospitalization. Severe LV dysfunction and free Tricuspid regurgitation have not heretofore been reported in the medical literature.
\end{abstract}

Keywords: Arrhythmogenic Right Ventricular Dysplasia, Cardiomyopathy, Atrial Fibrillation, Case Report, LV Dysfunction

\section{Introduction}

Being known as a rare genetic disorder, Arrhythmogenic Right Ventricular Dysplasia (ARVD) occurs due to the replacement of right ventricular myocardium with fibrofatty tis-sue that comes with ventricular arrhythmias and sudden cardiac death (SCD). The prevalence of ARVD is estimated between 1:2000 and 1:5000. It can lead to right ventricle (RV) volume increment and RV ejection fraction decrement. The ARVD condition is an essential trigger of ventricular arrhythmias in children and young adults, possibly leading to palpation, syncope, and even heart failure symptoms in advanced stages (1). The diagnosis is based on major and minor standardized task force criteria, a combination of clinical, morphological, and electrocardiographic aspects as detailed by the European Society of Cardiology (ESC) and the World Heart Federation (2). Tricuspid regurgitation (TR) and left ventricle dysfunction may occur due to right ventricle structural changes that are associated with worse prognosis (3). In this paper, we aim to cast light on clinical di-agnostic and treatment methods of the unique case of a nine-year-old boy diagnosed with ARVD with free TR and LV dysfunction.

\section{Case Presentation}

A nine-year-old boy was presented with generalized edema, ascites, and palpitation for the last two weeks. The patient was not febrile and did not have any history of chest pain, syncope, or dyspnea. Before this episode, he had been complaining of cough and fever, which recovered after taking antibiotics.

He had no significant family medical history. Holosystolic murmur of tricuspid regurgitation was detected on cardiac auscultation besides the presence of shifting dullness on abdominal examination. There were no other abnormalities on examination. The clinical signs and symptoms were in favor of chronic right-sided heart failure.

The blood count, routine biochemical screening, serum bicarbonate level, liver function tests, and viral PCR levels were within the normal ranges, but hypothyroidism was seen. The 12-lead electrocardiogram showed an inverted $\mathrm{T}$ wave in the precordial leads V1 - V3, atrial fibrillation, and epsilon wave. Echocardiography revealed severe right ventricle dysfunction, mild left ventricle dysfunction, severe RAE/RVE, free tricuspid regurgitation, and left ventricle ejection fraction of $45 \%$ - 50\% (Figures 1 and 2).

Cardiac magnetic resonance (CMR) with late enhance- 


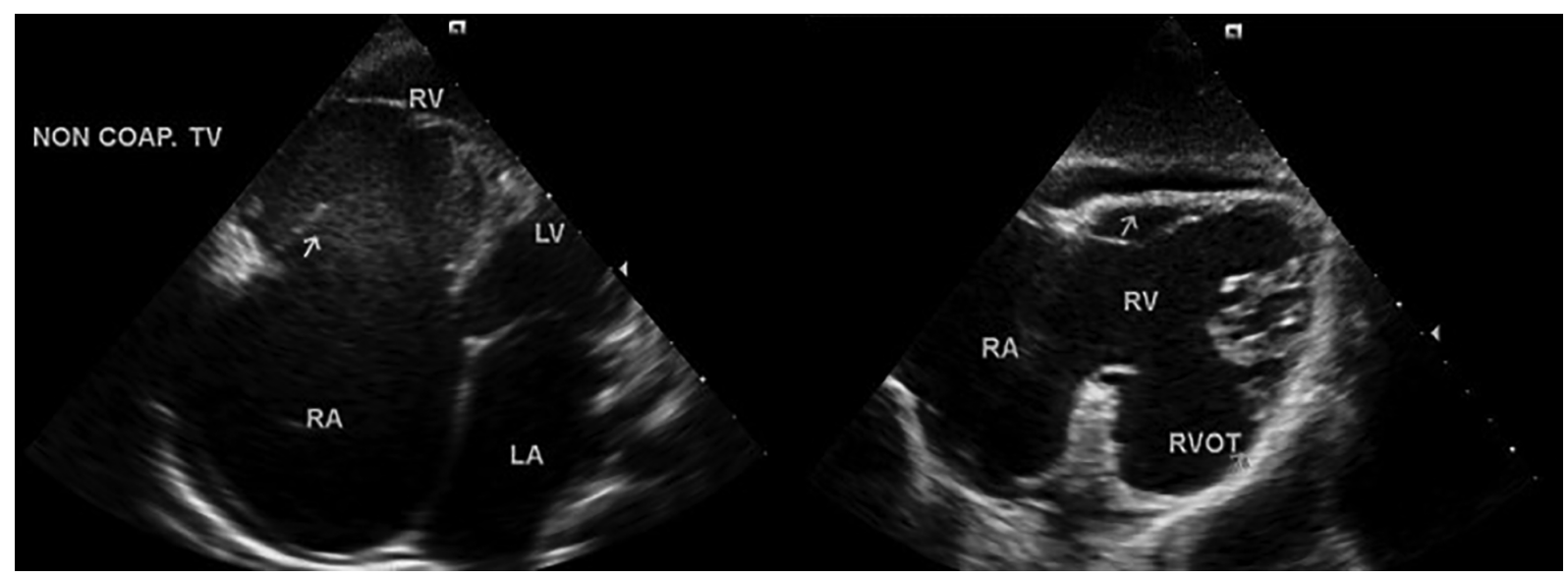

Figure 1. The echocardiographic examination indicating free tricuspid regurgitation, non-coaptative tricuspid valve and severe right atrial (RA) and right ventricular (RV) enlargement

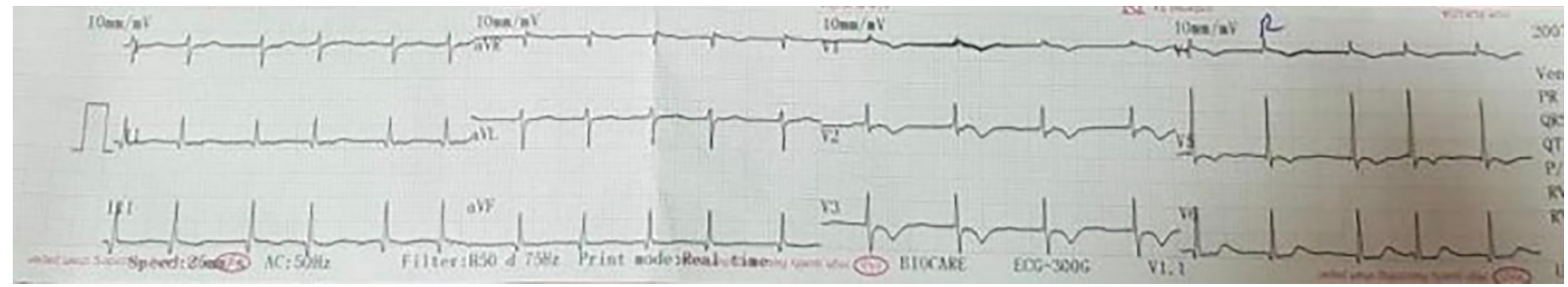

Figure 2. Electrocardiographic examination indicating atrial fibrillation

ment (LE) disclosed the findings that were consistent with the major criteria of arrhythmogenic right ventricular dysplasia including severely enlarged right ventricle size with severely reduced systolic function, dilated RVOT, akinesia in RVOT and RV free wall and left ventricle with moderately to severely reduced systolic dysfunction.

Moreover, 24-hour ECG Holter revealed normal diurnal variation with minimum and maximum HR of 48 and 174 b/min, respectively. Moreover, it showed PACs whole tracing. The QTc was $495 \mathrm{~ms}$. Genetic analysis was not done because all other criteria were fulfilled.

Prolonged episodes of sinus bradycardia were noted at each hospitalization. Overall, the patient met the criteria for ARVD. After the diagnosis, the patient received digoxin, furosemide, captopril, Aldactone, anticoagulant and antiplatelet agents (warfarin, ASA). Anti-arrhythmic agent (Amiodarone) was added to the treatment regimen since the inial event of atrial fibrillation.

\section{Discussion}

Arrhythmogenic Right Ventricular Dysplasia (ARVD) primarily affects the right ventricle. Several major and mi- nor criteria have been defined for the diagnosis of ARVD, including RV systolic dysfunction with the preservation of LV function on non-invasive cardiac imaging. The LV involvement may appear through the dysplastic process, hence leading to biventricular pump failure that is a significant risk predictor for sudden cardiac death (4).

There are many conflicts in LV involvement, including abnormalities of the LV wall motion, systolic dysfunction, and dilatation of the LV. In addition, atrial arrhythmias are associated with LV and RV involvement, especially arterial fibrillation. The prevalence of arterial arrhythmia in AVRD patients is about $14 \%$ that tricuspid regurgitation and RV dilation are the most important predicting factors; they appear to be suggestive for disease severity.

Tricuspid regurgitation (TR) is considered to be secondary to right-sided heart disease. The RV and right atrial (RA) remodeling may result in the presentation of TR in these patients. It may be conducive to aggravate heart failure by both RV filling pressure increment and RV stroke volume decrement (5). Our case was a nine-year-old boy whose AVRD was diagnosed based on epsilon wave and inverted T wave in V1 - V3 on ECG, RVOT aneurysm, RV EF of < 
$40 \%$ on MRI, PAC of more than 1000 on Holter, and RV FAC of $<40 \%$ on echocardiography. Free tricuspid and LV dysfunction were also reported in echocardiography. He also developed AF arrhythmia. To the best of our knowledge, this is the first AVRD case with free TR and LV dysfunction.

\section{Footnotes}

Conflict of Interests: The authors declare that they have no competing interests.

Funding/Support: This study was not funded by any grants.

Informed Consent: Informed consent was obtained from the patient.

\section{References}

1. Li KHC, Bazoukis G, Liu T, Li G, Wu WKK, Wong SH, et al. Arrhythmogenic right ventricular cardiomyopathy/dysplasia (ARVC/D) in clinical practice. J Arrhythm. 2018;34(1):11-22. doi: 10.1002/joa3.12021. [PubMed: 29721109]. [PubMed Central: PMC5828272].

2. Elias Neto J, Tonet J, Frank R, Fontaine G. Arrhythmogenic right ventricular cardiomyopathy/dysplasia (ARVC/D) - what we have learned after 40 years of the diagnosis of this clinical entity. Arq Bras Cardiol. 2019;112(1):91-103. doi: 10.5935/abc.20180266. [PubMed: 30673021]. [PubMed Central: PMC6317628].

3. Chungsomprasong P, Hamilton R, Luining W, Fatah M, Yoo SJ, GrosseWortmann L. Left ventricular function in children and adolescents with arrhythmogenic right ventricular cardiomyopathy. Am J Cardiol. 2017;119(5):778-84. doi: 10.1016/j.amjcard.2016.11.020. [PubMed: 28040191].

4. Stecker EC, Vickers C, Waltz J, Socoteanu C, John BT, Mariani R, et al. Population-based analysis of sudden cardiac death with and without left ventricular systolic dysfunction: Two-year findings from the Oregon Sudden Unexpected Death Study. J Am Coll Cardiol. 2006;47(6):1161-6. doi: 10.1016/j.jacc.2005.11.045. [PubMed: 16545646].

5. Pinamonti B, Dragos AM, Pyxaras SA, Merlo M, Pivetta A, Barbati $\mathrm{G}$, et al. Prognostic predictors in arrhythmogenic right ventricular cardiomyopathy: Results from a 10-year registry. Eur Heart J. 2011;32(9):1105-13. doi: 10.1093/eurheartj/ehr040. [PubMed: 21362707]. 